\title{
ON TWO EARLY UNIFIED FIELD THEORIES
}

\author{
A. H. KLOTZ \\ (Received 15 March 1968)
}

\section{Introduction}

In this article we shall compare two unified field theories namely, Einstein's first presentation of a four dimensional theory (refs. 1, 2) and Levi-Civita's reformulation of it (ref. 3). It is well known that the problem of a unified field theory (of macroscopic world) is to find a suitable entity within a proposed geometry to represent the electromagnetic field. Gravitation is usually built into the theory since most of theories utilising a four dimensional space time as a model of the physical world consist in a direct extension of General Relativity.

Although Einstein himself abandoned later his original choice of an electromagnetic vector potential, our remarks are prompted by two considerations. First, no unified field theory has ever been 'disproved'. In view of the numerous attempts to formulate one it is likely that if one should eventually be accepted, it will in some sense contain the ideas previously discussed. Secondly, we wish to consider the assertion that Einstein's and Levi Civita's theories are equivalent. This has been made by the latter Author and we may find a similar statement in a recent work of Mme Tonnelat (ref. 4, pp. 280-81).

Two theories may be regarded as equivalent either if they lead to the same field equations or if the basic geometry of the space time is the same in both. It is clear of course, that the latter sense does not apply in our case. Levi Civita's theory employs an ordinary Riemann geometry while Einstein's is, from the outset, non riemannian. We shall show below that the first equivalence does not hold either. At least the electromagnetic field is basically distinct in the two theories. Thus, any similarity between them derives only from the first approximation in which a wave equation for the field quantities is obtained in both.

\section{The $n$-uplet rotation}

We use Greek indices to refer to space-time coordinates and Latin ones to a lattice of lines which we assume can be constructed at any point of the manifold. The theory is formulated independently of whether the manifold 
is four-dimensional but if the geometrical space time is $n$-dimensional there are $n$ congruences at every point also. Thus both Greek and Latin indices go from 1 to $n$ (or, tacitly, from 1 to 4 ). The Greek, coordinate indices are raised or lowered with the help of a symmetric metric tensor but there is no corresponding operation for the $n$-uplet and Latin indices are shifted purely for notational convenience. Both Einstein and Levi-Civita set up an $n$-uplet (tetrad) in their manifold but Einstein uses it to define a nonsymmetric affine connection $\Gamma_{\mu \nu}^{\lambda}$ while Levi-Civita constructs with its help the Ricci coefficients of rotation $\gamma_{i j k}$.

We shall assume that both theories start with the same Riemannian manifold $V_{n}$. The $n$-uplets cannot then be identical for, with Einstein's connection, the $\gamma_{i j k}$ 's would vanish identically.

Let us denote Einstein's $n$-uplet parameters by $h_{\mu}^{i}$ and Levi-Civita's by $\lambda_{i / \mu}$. The stroke merely separates Greek and Latin indices and we use the semicolon notation for covariant derivatives. The summation convention applies to both kinds of indices. Then the metric tensor $g_{\mu \nu}$ is defined either by

or by

$$
g_{\mu \nu}=h_{\mu}^{a} h_{\nu}^{a}, \quad g^{\mu \nu}=h_{a}^{\mu} h_{a}^{\nu},
$$

$$
g_{\mu \nu}=\lambda_{a / \mu} \lambda_{a / \nu}, g^{\mu \nu}=\lambda_{a}^{\mu} \lambda_{a}^{\nu} .
$$

There exists a transformation

$$
d x_{a}^{\prime}=h_{a}^{\mu} d x_{\mu}, d x_{\mu}=h_{a}^{\mu} d x_{a},
$$

for which the metric is Cartesian in the ' $n$-uplet' frame. However, (2) must be non-integrable if the space is to remain curved.

We define, with Einstein, an affine connection, as

$$
\Gamma_{\mu \nu}^{\sigma}=-h_{\mu}^{a} h_{a, \nu}^{\sigma}=h_{a}^{\sigma} h_{\mu, \nu}^{a},
$$

whence

$$
g_{\mu \nu ; \lambda}=g_{\mu \nu, \lambda}-\Gamma_{\mu \lambda}^{\rho} g_{\rho \nu}-\Gamma_{\nu \lambda}^{\rho} g_{\mu \rho}=0 .
$$

Similarly

$$
\gamma_{i j \mu}=\lambda_{i / \mu ; \nu} \lambda_{j}^{\mu} \lambda_{k}^{\nu},
$$

so that (4) ensures its skew-symmetry in $i$ and $j$ :

$$
\gamma_{i j k}+\gamma_{j i k}=0 \text {. }
$$

If we identify the two metric tensors of (1) with each other, it follows that there exists a (local, that is, applying to the Latin, $n$-uplet indices) transformation

$$
\lambda_{i}^{\mu}=A_{i a} h_{a}^{\mu}, h_{\mu}^{a}=\lambda_{i j \mu} A_{i a}, h_{a}^{\mu}=\lambda_{i}^{\mu} A_{i a} .
$$


Clearly (under the assumption of orthogonality of the $n$-uplets), we have

$$
A_{i a}=\lambda_{i j \mu} h_{a}^{\mu}
$$

The transformation coefficients $A_{i a}$ form a representation of the rotation group which operates on the elements of the abstract space of local (that is, $n$-uplet or 'Latin' index) tensors. Indeed

$$
A_{i a} A_{j a}=\delta_{i j}=A_{a i} A_{a j} .
$$

A new form for the Ricci coefficients (5) is obtained if we use Einstein's connection (3).

Following Levi-Civita we introduce first invariant differentiation along the lattice lines. Thus

Then

$$
\frac{d}{d s_{k}}=\lambda_{k}^{\sigma} \frac{\partial}{\partial x_{\sigma}}, \quad \frac{\partial}{\partial x_{\mu}}=\lambda_{i / \mu} \frac{d}{d s_{i}} .
$$

$$
\begin{aligned}
\gamma_{i j k} & =\lambda_{i / \mu ; \nu} \lambda_{j}^{\mu} \lambda_{k}^{\nu}=\left(A_{i a}\right)_{; \nu} A_{j b} A_{k e} h_{b}^{\mu} h_{c}^{\nu} h_{\mu}^{a} \\
& =A_{e c} A_{j a} A_{k b} \frac{d A_{i a}}{d s_{e}} h_{\nu}^{c} h_{b}^{\nu} \\
& =A_{j a} \frac{d A_{i a}}{d s_{k}}
\end{aligned}
$$

since local quantities are space-time invariants. The fact that the Ricci coefficients can be expressed exclusively in terms of the $A$-transformation coefficients and their lattice-derivatives, plays a large part in our proof of non-equivalence of Einstein's and Levi-Civita's theories.

\section{The electromagnetic field tensor}

Levi Civita assumes the localised electromagnetic field tensor $f_{i j}$ to be proportional to the space invariant

$$
\zeta_{i j}=\frac{d \gamma_{i j k}}{d s_{k}}
$$

The constant of proportionality is a numerical multiple of an elementary electric charge. On the other hand, Einstein identifies

$$
\Gamma_{\mu}=2 \Gamma_{\mu \sigma}^{\sigma}=\Gamma_{\mu \sigma}^{\mu}-\Gamma_{\sigma \mu}^{\sigma}=h_{a}^{\sigma}\left(h_{\mu, \sigma}^{a}-h_{\sigma, \mu}^{a}\right),
$$

with the electromagnetic potential vector. His field tensor, $F_{i j}$ say (in localised form) is therefore

$$
F_{i j}=\lambda_{i}^{\mu} \lambda_{j}^{\nu}\left(\Gamma_{\mu, \nu}-\Gamma_{\nu, \mu}\right) .
$$


It is not clear yet whether (12) and (14) can not be reconciled. We prove first that $\Gamma_{\mu}$ can be defined equivalently from either theory.

Indeed, from the definition (5) of a Ricci coefticient, we have

whence

$$
2 \Gamma_{\mu \nu}^{\sigma}=\lambda_{i}^{\sigma}\left(\lambda_{i / \mu, \nu}-\lambda_{i / \nu, \mu}-\left(\gamma_{i j k}-\gamma_{i k j}\right) \lambda_{j / \mu} \lambda_{k / \nu}\right),
$$

$$
\Gamma_{\mu}=\lambda_{i}^{\sigma}\left(\lambda_{i / \mu, \sigma}-\lambda_{i / \sigma, \mu}\right)-\gamma_{i j i} \lambda_{j / \mu} .
$$

But, from (3) using the rotation (7), and equation (11)

Since

$$
2 \Gamma_{\mu \nu}^{\sigma}=\lambda_{i}^{\sigma}\left(\lambda_{i / \mu, \nu}-\lambda_{i / \nu, \mu}\right)+\lambda_{i}^{\sigma} \gamma_{j i k}\left(\lambda_{j / \mu} \lambda_{k / \nu}-\lambda_{j / \nu} \lambda_{k / \mu}\right) .
$$

$$
\gamma_{i i j}=0 \text {, }
$$

a contraction of (16) leads back to (15) and this proves our assertion.

It follows that we can use either definition of $\Gamma_{\mu}$ to calculate Einstein's, localised electromagnetic tensor. In fact a simple reduction gives

Since

$$
\begin{aligned}
F_{i j}= & -\left(\lambda_{i}^{\mu} \lambda_{j}^{\nu}-\lambda_{i}^{\nu} \lambda_{j}^{\mu}\right)\left(\lambda_{k, \nu}^{\sigma} \lambda_{k / \sigma, \mu}+\lambda_{k, \nu}^{\sigma} \lambda_{k / \mu, \sigma}\right. \\
& \left.+\lambda_{k / \mu} \lambda_{k, \nu \sigma}^{\sigma}+\gamma_{k l k} \lambda_{k / \mu, \nu}\right)-\frac{d}{d s_{j}} \lambda_{k i k}+\frac{d}{d s_{i}} \gamma_{k j k} .
\end{aligned}
$$

and

$$
\lambda_{k / \sigma, \mu}=\gamma_{k m n} \lambda_{m / \sigma} \lambda_{n / \mu}+\Gamma_{\sigma \mu}^{\tau} \lambda_{k / \tau}
$$

we have

$$
\lambda_{k, \nu}^{\sigma}=\gamma_{k m n} \lambda_{m}^{\sigma} \lambda_{n / \nu}-\Gamma_{\tau \nu}^{\sigma} \lambda_{k}^{\tau},
$$

$$
\begin{aligned}
\lambda_{k / \mu, \nu \sigma}= & \gamma_{k m n}\left(\gamma_{m \rho q} \lambda_{\rho / \mu} \lambda_{\alpha / \sigma} \lambda_{n / \nu}+\gamma_{n \rho q} \lambda_{\rho / \nu} \lambda_{q / \sigma} \lambda_{m / \mu}\right. \\
& \left.+\Gamma_{\mu \sigma}^{\rho} \lambda_{n / \nu} \lambda_{m / \rho}+\Gamma_{\nu \sigma}^{\rho} \lambda_{n / \rho} \lambda_{m / \mu}\right) \\
& +\Gamma_{\mu \nu}^{\rho}\left(\gamma_{k \rho q} \lambda_{p / \rho} \lambda_{q / \sigma}+\Gamma_{\sigma}^{\tau} \lambda_{k / \tau}\right)+\Gamma_{\mu \nu, \sigma} \lambda_{k /} \\
& +\lambda_{r / \sigma} \lambda_{m / \mu} \lambda_{n / \nu} \frac{d}{d s_{r}}\left(\gamma_{k m n}\right) .
\end{aligned}
$$

Using these expressions we find that

where

$$
\begin{aligned}
F_{i j}= & \mathscr{E}_{i j}+\frac{d}{d s_{k}}\left(\gamma_{k i j}-\gamma_{k j i}\right)-\frac{d}{d s_{j}} \gamma_{k i k}+\frac{d}{d s_{i}} \gamma_{k j k} \\
& +2 \lambda_{i}^{\mu} \lambda_{j}^{\nu} \Gamma_{\mu \nu ; \sigma}^{\sigma}+2 \gamma_{k_{m n}} \Gamma_{\mu \sigma} \lambda_{k}^{\sigma}\left(\lambda_{m /} \lambda_{i}^{\mu} \delta_{j n}\right. \\
& \left.-\lambda_{m /} \lambda_{j}^{\mu} \delta_{i n}-\lambda_{m /} \lambda_{i}^{\mu} \delta_{j m}+\lambda_{n /} \lambda_{j}^{\mu} \delta_{i m}\right),
\end{aligned}
$$

$$
\begin{aligned}
\mathscr{E}_{i k}= & \left(\delta_{i p} \delta_{j n}-\delta_{i n} \delta_{j p}\right)\left(\gamma_{k m n} \gamma_{m p k}+\gamma_{k p m} \gamma_{p m n}\right. \\
& \left.+\gamma_{k m n} \gamma_{k p m}+\gamma_{m k n} \gamma_{k m p}\right) .
\end{aligned}
$$


Furthermore, if we introduce Levi-Civita's four index symbols

$$
\gamma_{i j, h k}=\frac{d_{\gamma i j n}}{d s_{k}}-\frac{d_{\gamma_{i j k}}}{d s_{h}}+\gamma_{i j l}\left(\gamma_{l h k}-\gamma_{l k h}\right)+\gamma_{l i h} \gamma_{l j h}-\gamma_{l i h} \gamma_{l j k},
$$

we find that

$$
\mathscr{E}_{i j}+\frac{d}{d s_{k}}\left(\gamma_{k i j}-\gamma_{k j i}\right)-\frac{d}{d s_{j}} \gamma_{k i k}+\frac{d}{d s_{i}} \gamma_{k j k}=\gamma_{k i, j k}-\gamma_{k j, i k}=0,
$$

whence finally

$$
\frac{1}{2} F_{i j}=\gamma_{i}^{\alpha} \lambda_{j}^{\beta} \Gamma_{\alpha \beta ; \sigma}^{\sigma}+\Gamma_{\mu \sigma}^{\rho} \lambda_{m / \rho} \lambda_{k}^{\sigma}\left(\delta_{j n} \lambda_{i}^{\mu}-\delta_{i n} \lambda_{j}^{\mu}\right)\left(\gamma_{k m n}-\gamma_{k n m}\right) .
$$

\section{Conclusions}

We can deduce now easily that the theories of Einstein and of LeviCivita are incompatible. Let us for example, choose

$$
\lambda_{i}^{\mu}=h_{i}^{\mu}
$$

as a special case. Then, as mentioned above, the Ricci coefficients vanish identically. However, Einstein's field tensor as given by (17) becomes

$$
F_{i j}=\lambda_{i}^{\alpha} \lambda_{j} \Gamma_{\alpha \beta ; \sigma}^{\sigma}
$$

and is non zero. In Levi-Civita's theory however, this case corresponds to an absence of electromagnetic fields.

\section{Summary}

It is shown that Einstein's 1929 Unified Field Theory and that of Levi-Civita are incompatible.

\section{References}

[1] A. Einstein, Berl. Ber. 1928.

[2] A. S. Eddington, Nature. 123, 280, 1929.

[3] T. Levi-Civitta, 'A Simplifjed Presentation of Einstein's Unified Field Equations', London and Glasgow 1950.

[4] M. A. Tonnelat 'Les Théories Unitaires de L'Électromagnétisme et de La Gravitation', Paris 1965.

University of Sydney

Sydney, NSW 